\title{
To Construct an Engineered (S)-Equol Resistant E. coli for in Vitro (S)-Equol Production
}

OPEN ACCESS

Edited by:

Mattheos Koffas,

Rensselaer Polytechnic Institute,

United States

Reviewed by:

Haoran Zhang,

Rutgers, The State University of New Jersey, United States

Gyoo Yeol Jung,

Pohang University of Science and Technology, South Korea

*Correspondence:

Xin Wang

xxww101@sina.com Yeshi Yin

yinyeshi@126.com

tThese authors have contributed equally to this work.

Specialty section:

This article was submitted to Microbial Physiology and Metabolism, a section of the journal Frontiers in Microbiology

Received: 22 February 2018 Accepted: 15 May 2018 Published: 04 June 2018

Citation:

Li H, Mao S, Chen H, Zhu L, Liu W, Wang $X$ and Yin $Y$ (2018) To Construct an Engineered (S)-Equol Resistant E. coli for in Vitro (S)-Equol Production. Front. Microbiol. 9:1182. doi: 10.3389/fmicb.2018.01182

\begin{abstract}
Hailiang $\mathrm{Li}^{1,2,3+}$, Shaoming Mao ${ }^{3 \dagger}$, Huahai Chen ${ }^{1,2}$, Liying $\mathrm{Zhu}^{2}$, Wei Liu${ }^{2}$, Xin Wang ${ }^{2 *}$ and Yeshi Yin ${ }^{1,2 *}$

' Key Laboratory of Comprehensive Utilization of Advantage Plants Resources in Hunan South, College of Chemistry and Bioengineering, Hunan University of Science and Engineering, Yongzhou, China, ${ }^{2}$ State Key Laboratory Breeding Base for Zhejiang Sustainable Pest and Disease Control, Institute of Plant Protection and Microbiology, Zhejiang Academy of Agricultural Sciences, Hangzhou, China, ${ }^{3}$ Hunan Provincial Key Laboratory for Forestry Biotechnology, College of Life Science and Technology, Central South University of Forestry and Technology, Changsha, China
\end{abstract}

(S)-equol is one of the major metabolites of daidzein that is produced by human and animal gut bacteria. Most of the physiological functions of soybean isoflavones, such as anti-oxidative activity, anti-cancer activity, and cardiovascular protection have been ascribed to (S)-equol. However, only 30-50\% people contain this kind of equol-producing bacteria, and therefore are able to convert daidzein to (S)-equol. Administration of (S)-equol may be more beneficial than soybean isoflavones. The aim of this study was to construct an engineered (S)-equol resistant Escherichia coli to enhance (S)-equol production in vitro. First, transposon mutagenesis libraries were constructed and screened to isolate the (S)-equol resistant mutant E. coli strain BL21 (ydiS) in order to overcome the inhibitory effects of $(S)$-equol on bacterial growth. Bacterial full genome scan sequencing and in vitro overexpression results revealed that the ydis gene was responsible for this resistance. Second, the (S)-equol-producing genes Ldznr, L-ddrc, L-dhdr, and L-thdr of Lactococcus strain 20-92 were synthesized and cloned into compatible vectors, pETDuet-1 and pCDFDuet-1. These plasmids were subsequently transformed into BL21 (DE3) and its mutant BL21 (ydiS). Both engineered BL21 (DE3) and BL21 (ydiS) could use daidzein as substrate to produce (S)-equol under both anaerobic and aerobic conditions. As expected, engineered BL21 (ydiS) had faster growth rates than BL21 (DE3) when supplemented with high concentrations of $(S)$-equol. The yield and the daidzein utilization ratio were higher for engineered BL21 (ydiS). Interestingly, engineered BL21 (ydiS) was able to convert daidzein to $(S)$ equol efficiently under aerobic conditions, providing a convenient method for (S)-equol production in vitro. In addition, a two-step method was developed to produce (S)-equol using daidzin as substrate.

Keywords: (S)-equol production, (S)-equol resistance, soybean isoflavone, transposon mutagenesis, ydiS gene

\section{INTRODUCTION}

Soy isoflavones have multiple health benefits due to their anti-carcinogenic, anti-oxidant, and anti-atherosclerotic properties (Xiao et al., 2017). These chemicals also interact with the estrogen receptor, enabling them to act as weak to moderate phytoestrogens (Nielsen and Williamson, 2007). Interestingly, a variety of studies have suggested that the clinical effectiveness of isoflavones might 
be due to their metabolites (Setchell et al., 2002; Sarkar and Li, 2003; Cobb et al., 2006; Cooke, 2006; Jackman et al., 2007). In 2002, Setchell et al. (2002) proposed the "Equol Hypothesis," which posits that daidzein is converted to $(S)$-equol by gut bacteria in certain individuals, and that it is the equol that accounts for the noted health benefits of soy isoflavones.

Equol (7-hydroxy-3-[4-hydroxyphenyl]-chroman) was first isolated from equine urine in 1932 (Marrian and Haslewood, 1932) and was also identified 50 years later in human urine as a metabolite of soy isoflavones (Axelson et al., 1982). Equol is not present in soybeans, but it is produced naturally in the human gut by daidzein, a major isoflavone predominantly found in soybean, by intestinal bacteria (Setchell and Clerici, 2010). Equol exhibits stronger anti-oxidant and estrogenic activities than daidzein (Hwang et al., 2003; Kinjo et al., 2004; Turner et al., 2004; Rufer and Kulling, 2006) and has been demonstrated to act as vasorelaxant (Jackman et al., 2007), along with having anti-inflammatory properties (Blay et al., 2010), which have both been observed previously in soy isoflavones (Chacko et al., 2005; Hall et al., 2008). However, only $30-50 \%$ of the human population can produce equol (Low et al., 2005; Ozasa et al., 2005; Setchell and Cole, 2006; Hall et al., 2007). This suggests that health effects of functional foods supplemented with $(S)$-equol could be more beneficial than daidzein. Several studies have demonstrated that a diet supplemented with natural $(S)$-equol alleviates menopausal symptoms, such as hot flushes and crow's feet wrinkles (Aso et al., 2012; Oyama et al., 2012).

Currently, the majority of equol production is performed by chemical synthesis, although production of $(S)$-equol via bacterial fermentation may have several advantages over chemical synthesis. Specific intestinal bacteria are responsible for the conversion of daidzein to $(S)$-equol, such as Coriobacteriaceae sp. and Lactobacillus sp. (Setchell and Clerici, 2010). The Hishigaki group has cloned and identified a gene cluster responsible for converting daidzein to (S)-equol from an equol-producing strain Lactococcus 20-92 (Shimada et al., 2011, 2012). The gene product of L-dznr is responsible for converting daidzein into $(R)$-dihydrodaidzein; the L-ddrc gene product converts $(R)$-dihydrodaidzein into $(S)$-dihydrodaidzein; the L-dhdr gene product converts (S)-dihydrodaidzein into trans-tetrahydrodaidze; and the L-thdr gene product converts trans-tetrahydrodaidzein into (S)-equol (Shimada et al., 2011, 2012). Lee et al. (2016) constructed a recombinant Escherichia coli BL21 strain which can produce (S)-equol in vitro. However, Vázquez et al. (2017) reported that isoflavone-derived compounds like $(S)$-equol have the ability to inhibit the growth from many bacteria species. The aim of this study was to obtain an $(S)$-equol resistant host $E$. coli, which can be engineered for $(S)$-equol production by co-expressing the equol-producing genes L-ddrc, L-dznr, L- $d h d r$, and L-thdr. As a result, a putative oxidoreductase gene $y d i S$ was identified to be responsible for the $(S)$-equol resistance. An engineered equol-producing bacterial strain was constructed using an $(S)$-equol resistant mutant [E. coli BL21 ( $y$ diS)] to coexpress the equol-synthesis genes. A two-step method was utilized to convert diadzin to $(S)$-equol under aerobic conditions. All results of this study have been summarized in a schematic diagram (Figure 1).

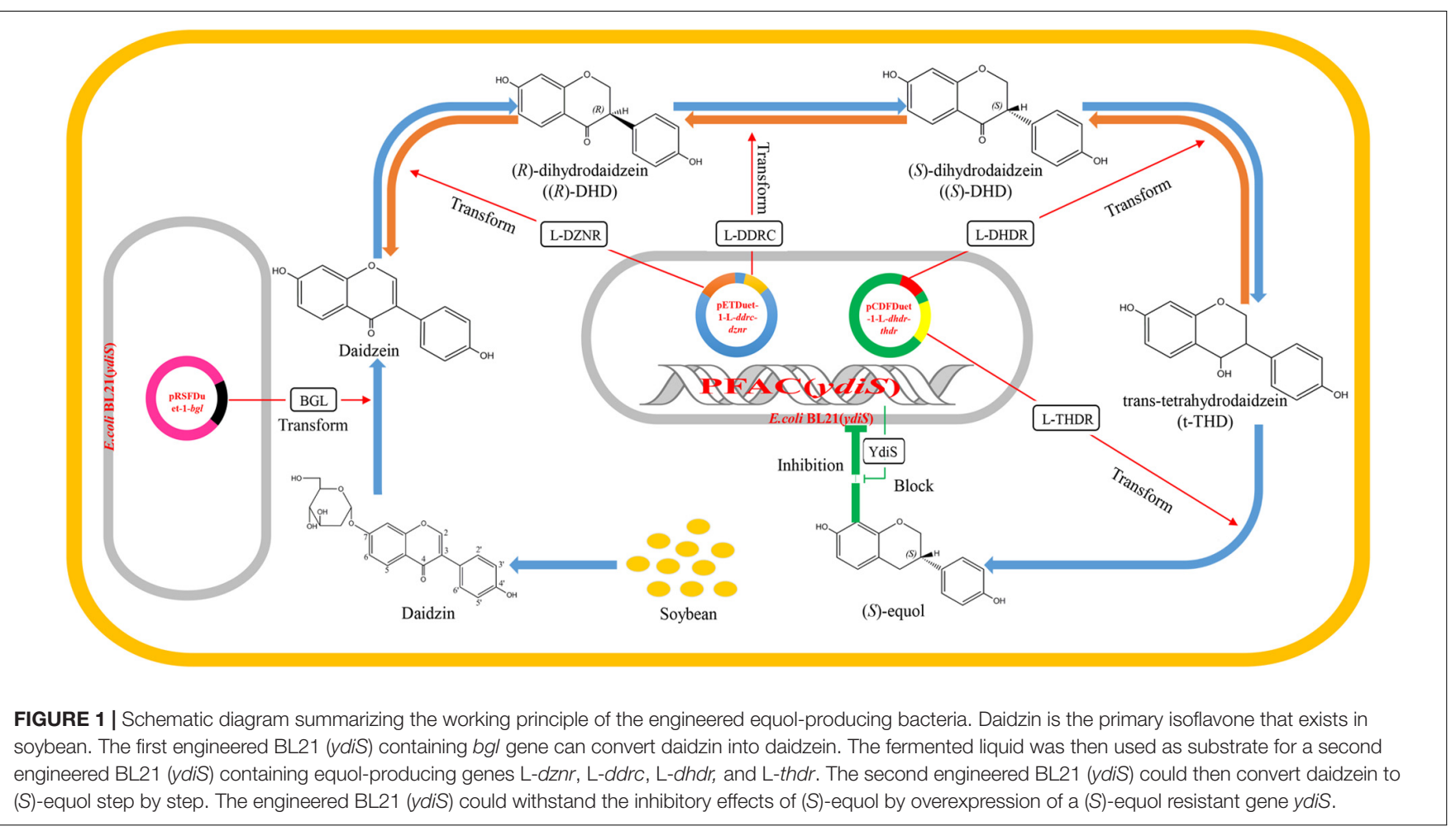




\section{MATERIALS AND METHODS}

\section{Chemicals and Reagents}

Daidzin, daidzein and $(S)$-equol were purchased from Daicel Chiral Technologies Co., Ltd. (Shanghai, China). The antibiotics and isopropyl-D-thiogalatopyranoside (IPTG) were ordered from Sangon Biotech Bio (Shanghai, China). The restriction enzymes and ligation kit were purchased from TaKaRa Bio (Dalian, China).

\section{Bacteria Strains, Plasmids, and Growth Conditions}

Detailed information regarding the strains and plasmids used in this study is listed in Supplementary Table 1. In brief, E. coli strains $\mathrm{DH} 5 \alpha$ and BL21 (DE3) were ordered from TaKaRa Bio (Dalian, China) and Transgene Biotech (Beijing, China), respectively. Mariner transposon plasmid pFAC (Wong and Mekalanos, 2000) and a dap auxotroph E. coli strain WM3064 (Saltikov and Newman, 2003) were obtained from Dr. Gao's laboratory (Zhejiang University, Hangzhou, China). DH5a E. coli (pRK2013) was ordered from Biomedal S. L. ${ }^{1}$. The genes L-dznr (GenBank accession number: AB558141.1), L-ddrc (GenBank accession number: AB694972.1), L-dhdr (GenBank accession number: AB592970.1), L-thdr (GenBank accession number: AB592969.1), and bgl (GenBank accession number: JQ957567.1) were synthesized and then sub-cloned the pUC57 vector by GenScript Biotechnology (Nanjing, China). The ydiS gene was PCR amplified using primers ydiS-F: 5-ATG TCG GAT GAC AAA TTT GAT GCC A-3, and $y$ dis-R: 5-ATC GCG CCA ACG AGG GAA TTA-3. The $y$ diT gene of BL21 (ydiS) was synthesized and sub-cloned into the pRSFDuet-1 vector by GenScript Biotechnology (Nanjing, China). E. coli compatible vectors pRSFDuet-1, pETDuet-1, and pCDFDuet-1 were acquired from Merck Millipore (Germany). E. coli strains were grown at $37^{\circ} \mathrm{C}$ in Lennox broth (LB) or LB agar. When required, $50 \mu \mathrm{g} / \mathrm{mL}$ carbenicillin, $50 \mu \mathrm{g} / \mathrm{mL}$ streptomycin, and $15 \mu \mathrm{g} / \mathrm{mL}$ kanamycin were added to the broth or plates. When required, an anaerobic chamber (anaerobic workstation AW 500, Electrotek Ltd., United Kingdom) was employed to minimize oxygen exposure.

\section{Transposon Mutagenesis Library Screening}

In this study, the transposon of the pFAC plasmid, consisting of a transposable element flanked by two inverted repeats of 27 bps ( $5^{\prime}$-aca ggt tgg ctg ata agt ccc cgg tct- $\left.3^{\prime}\right)$ and a gentamycin resistance cassette in the middle (aacC1: $534 \mathrm{bp}$ ) was used. A gene encoding the hyperactive mariner transposase, and a gene encoding $\beta$-lactamase (bla) were included in this plasmid (Withers et al., 2014). In theory, the promotor of gentamycin $\left(\mathrm{P}_{\mathrm{Gm}}\right)$ transferred together with the mariner transposon, causing adjacent genes to be overexpressed or repressed, dependent on the transcript directions for the gentamycin promotor and its downstream genes. Transposon mutagenesis was prepared via

${ }^{1}$ http://lifescience.biomedal.com/ conjugation utilizing pFAC plasmid-carrying E. coli WM3064 as the donor strain and BL21 (DE3) as the recipient strain. Transfer of plasmids from WM3064 to BL21 (DE3) were performed via tripartite conjugations using the helper plasmid pRK2013. In brief, bacterial E. coli WM3064, BL21 (DE3), and DH5 $\alpha$ (pRK2013) were incubated in LB media at $37^{\circ} \mathrm{C}$ overnight, $500 \mu \mathrm{L}$ of each bacterium was then mixed together in a $2 \mathrm{~mL}$ tube. After centrifuging, the bacterial pellet was resuspend using LB media and transferred onto a dry LB plate (supplemented with 2,6-diaminopimelic acid) in three compact droplets. After incubation for $\sim 6 \mathrm{~h}$, the bacteria were gathered and streaked onto LB plates supplemented with gentamicin $(15 \mu \mathrm{g} / \mathrm{mL})$. Bacterial colonies were then seeded into 96-well plates containing LB media supplemented with $200 \mu \mathrm{g} / \mathrm{mL}$ (S)-equol. The $\mathrm{OD}_{600}$ was detected using a Model 680 microplate reader (Bio-Rad, United States) and SP-2000UV spectrometer (Shanghai Spectrum Instruments Co., Ltd.). The equol-resistant character of the five clones was further verified using a tube culture method. Chromosomal DNA of these (S)-equol resistant mutants was isolated using an OMEGA Genomic DNA Extraction Kit (Omega, United States). Taxa identification was performed using by $16 \mathrm{~s}$ rRNA sequencing and BLAST analysis. The mutant $E$. coli strain BL21 ( $y$ diS) was then selected for full genome sequencing using Illumina Hiseq at Majorbio Bio-Pharm Technology Co., Ltd., Shanghai, China. The draft genome sequence data of BL21 (ydiS) has been deposited in NCBI as Accession Number PIYU00000000.

\section{Detection of Bacterial Growth Rate}

For static culture, $30 \mathrm{~mL}$ of LB media were added to a $100 \mathrm{~mL}$ flask bottle. After autoclaving, bacteria, antibiotics and chemical reagents were then added into the bottle. Bacterial density $\left(\mathrm{OD}_{600}\right)$ was measured every $3 \mathrm{~h}$. For culturing under shaking conditions, a real-time detection instrument Microscreen-16 (Gering Instrument Manufacturing (Tianjin) Co., Ltd., Tianjin, China) was used. $40 \mathrm{~mL}$ of LB media were added to the $50 \mathrm{~mL}$ measure bottle, and $400 \mathrm{rpm}$ (equivalent to $100 \mathrm{rpm}$ in the general shake incubator) was utilized for stirring. The optical absorption value was measured at 30-min intervals. Optical absorption was detected at $\mathrm{OD}_{850}$, and a conversion factor between $\mathrm{OD}_{850}$ and $\mathrm{OD}_{600}$ was calculated using E. coli before the experiment.

\section{Batch Culture Fermentation for Equol Production}

Batch culture fermentations were cultured without shaking at $37^{\circ} \mathrm{C}$, under both anaerobic and aerobic conditions. Briefly, a basic growth medium, LB (Qingdao Hope Bio-Technology Co., Ltd., Qingdao, China), was used to assess the utilization of daidzin or daidzein in the engineered equol-producing $E$. coli. For fermentation, bacteria were aliquoted into $50 \mathrm{~mL}$ flask bottles containing $20 \mathrm{~mL}$ of culture media supplemented with either daidzin or daidzein. Twenty microliters of IPTG (25 $\mathrm{mg} / \mathrm{mL}$ ) was added to each bottle to induce gene expression. Samples then were collected at 48 and $72 \mathrm{~h}$ after IPTG induction to detect equol and daidzein via HPLC. For production of 
$(S)$-equol from daidzin, a two-step fermentation was attempted in this study. For the first step, daidzin was transformed to daidzein using E. coli (pRSFDuet-1-bgl) under fermentation $72 \mathrm{~h}$. The fermentation liquid was then collected after centrifugal separation, and the upper liquid was used for preparing a new LB media (LB-D). For the second step, DDDT-BL21 (ydis) was inoculated into the LB-D media to detect the equol production.

\section{HPLC Detection}

Identification of equol and daidzein was performed using HPLC according to a previously described method with some modification (Decroos et al., 2005). In brief, $1 \mathrm{~mL}$ of each sample was extracted three times with $1 \mathrm{~mL}$ acidic ether, then the ether fractions were combined, evaporated to dryness and resuspended in $200 \mu \mathrm{L}$ of methanol and stored at $-20^{\circ} \mathrm{C}$ until analysis. HPLC analysis was performed using a Waters e2695 system. Fifteen microliter aliquots of each sample were injected and separated using a SunFireTM C18 $5 \mu \mathrm{m}$ column $(4.6 \mathrm{~mm} \times 205 \mathrm{~mm})$. The temperature was set at $30 \pm 2{ }^{\circ} \mathrm{C}$ and the flow rate was maintained at $0.8 \mathrm{~mL} / \mathrm{min}$. Elution was isocratic with a mobile phase consisting of $0.01 \%$ formic acid:methanol:acetonitrile (50:20:30). Equol was detected at $205 \mathrm{~nm}$; daidzein at $254 \mathrm{~nm}$. Calibration curves for the quantification of daidzein and equol were constructed using pure standards obtained from Daicel Chiral Technologies Co., Ltd. (Shanghai, China).

\section{Statistical Analysis}

SPSS Software (version 20.0; SPSS Inc., United States) and the Student's $t$-test was employed in this study. $P<0.05$ was considered to be statistically significant.

\section{RESULTS}

\section{Inhibitory Effects of (S)-Equol on Host E. coli BL21 (DE3)}

Previously, (S)-equol was shown to inhibit the growth of representative human gut bacteria (Vázquez et al., 2017). However, the inhibitory effects of the fermentation product (S)-equol on host bacterial E. coli BL21 (DE3) requiring further investigation. In this study, an E. coli strain was engineered to coexpress the four equol-producing genes L-ddrc, L-dznr, $\mathrm{L}-d h d r$, and L-thdr, which originated from an equol-producing bacterial Lactococcus strain 20-92 (Supplementary Figure 1). In order to evaluate the equol-producing activity of the engineered E. coli, $50 \mu \mathrm{g} / \mathrm{mL}(\sim 200 \mu \mathrm{M})$ daidzein was added to LB culturing media and $(S)$-equol production was detected under both anaerobic and aerobic conditions at different time points after IPTG induction. As demonstrated in Supplementary Figures $2 \mathrm{~A}, \mathrm{D}$, the metabolites from the engineered $E$. coli had a similar HPLC peaks to the reference standard for (S)equol. The LC-MS results further verified that the equol peak detected by HPLC had the same molecular weight as the (S)equol reference standard (Supplementary Figures 2B,C,E,F). Previous studies have reported that the equol produced by gut bacteria is (S)-equol (Setchell et al., 2005), and that the metabolite produced by Lactococcus strain 20-92 is ( $S$ )-equol (Shimada et al., 2011, 2012), the metabolite produced by the engineered E. coli was likely to be (S)-equol (Supplementary Figure 3). However, bacterial growth rates were inhibited during fermentation (Supplementary Figure 4); bacterial density decreased 30 and $37 \%$ under anaerobic and aerobic conditions, respectively, after IPTG was added as an inducer for $24 \mathrm{~h}$. In addition, there was almost no bacterial growth observed even cultured for $72 \mathrm{~h}$ (Supplementary Figure 4). In order to clarify which compounds had inhibitory effects on the growth of BL21 (DE3), bacterial plates were prepared using different compounds. The fermentation product $(S)$-equol did inhibit BL21 (DE3) growth both under both anaerobic and aerobic conditions (Supplementary Figure 5), however, daidzein did not cause inhibition. In order to further verify the inhibitory effects of daidzein and equol on bacterial growth, static liquid culture and shake culture experiment were done under aerobic conditions at $37^{\circ} \mathrm{C}$. Supplementary Figure 6 illuminated that equol has the ability to inhibit BL21 (DE3) growth which was dependent on the equol concentration. However, daidzein not inhibit growth and may have slightly promoted bacterial growth for both BL21 (DE3) and BL21 (G2) (Supplementary Figures 6A,B).

\section{Screening of (S)-Equol Resistant BL21 (ydiS) Mutants}

The feedback inhibitory effects of the fermentation products could prevent high yields of (S)-equol product, transposon mutagenesis libraries were constructed and screened in order to develop (S)-equol resistant bacteria. In summary, thounds of bacterial clones grown on $\mathrm{LB}+\mathrm{Gm}$ plates from the mutant library. 93 clones were then randomly picked up and then seeded in a 96-well plate to evaluate their equol resistance. The growth rate was monitored at different time points using a microplate reader. Among screened clones, five clones were identified as growing faster than BL21 (DE3) in the presence of $200 \mu \mathrm{g} / \mathrm{mL}$ (S)-equol (data not shown). Twenty-milliliter tubes containing $5 \mathrm{~mL}$ of LB and $5 \mu \mathrm{L}$ of $(S)$-equol were further used to verify the equol-resistance of these clones (Supplementary Figure 7). $16 \mathrm{~s}$ rRNA gene sequencing and BLAST analysis were then used for taxonomy analysis of these clones. Mutant E. coli strain BL21 (DE3)_G2 [renamed as BL21 (ydiS)] was selected for further verification and study. The bacterial density of BL21 (ydiS) was higher than BL21 (DE3) under both static and shaken culture conditions in the presence of 50 or $100 \mu \mathrm{g} / \mathrm{mL}(S)$-equol (Figure 2). This BL21 (ydiS) strain was then selected for equol production and has been deposited into the China General Microbiological Culture Collection Center (CGMCC No. 14219).

\section{Identification of Equol Resistant Gene in BL21 (ydiS)}

In order to identify the transposon insert site and infer the equol resistant mechanism of BL21 ( $y$ diS), full genome scanning and reverse PCR sequencing were performed. The sequencing results revealed that the PFAC transposon was inserted at $307 \mathrm{bp}$ upstream of the $y$ diS gene. The direction 

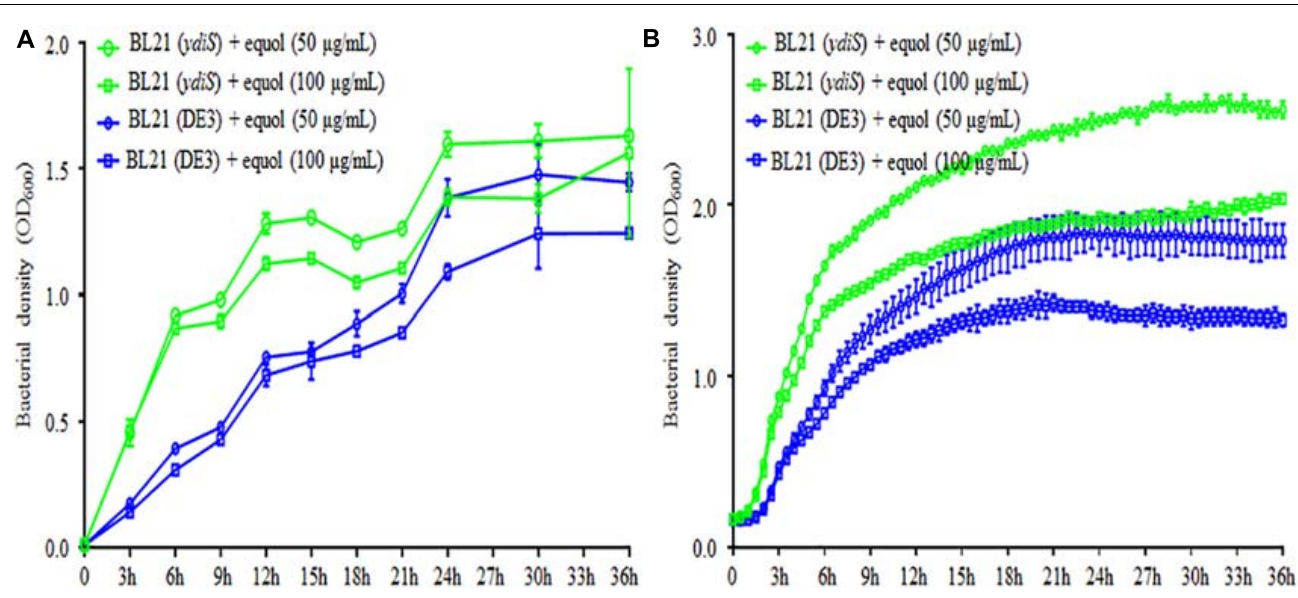

FIGURE 2 | (S)-equol resistance of BL21 (DE3) mutant. Growth rates under static (A) and shaking (B) culture conditions for the BL21 (DE3) and mutant BL21 (ydiS) were compared when different concentration of $(S)$-equol were added. At each timepoint, two duplications were measured for each sample, means and standard deviations (SD) were calculated.

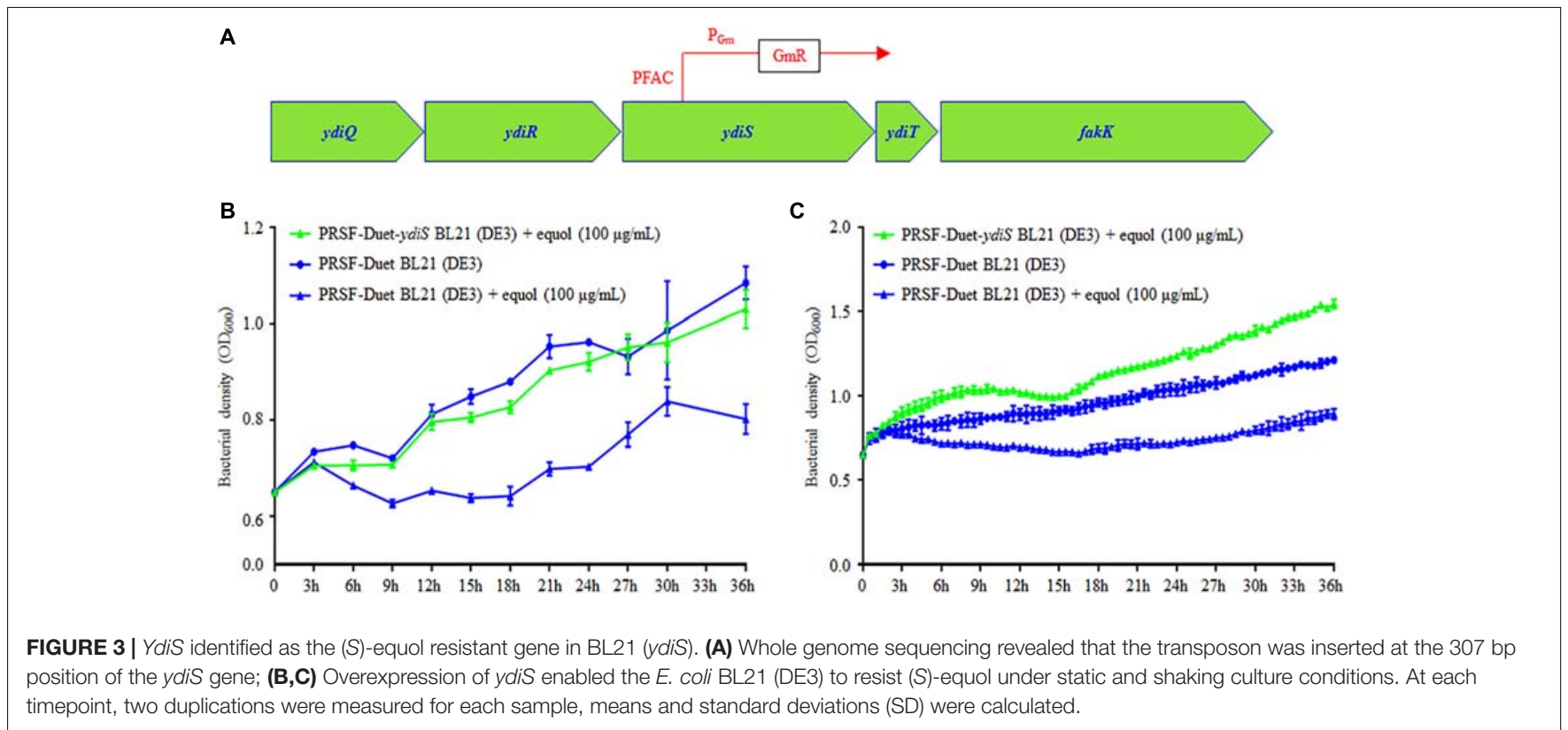

of PFAC Gm promotor is same as the $y$ diS gene (Figure $3 \mathrm{~A}$ ). Overexpression of $y d i S$ and its downstream gene $y$ diT may have contributed to the equol resistance for BL21 (ydiS). YdiS and $y$ diT genes were then cloned into pRSFDuet-1, respectively. When the $\mathrm{OD}_{600}$ reached $\sim 0.6,5 \mu \mathrm{L}$ of IPTG $(25 \mathrm{mg} / \mathrm{mL})$ was then added to induce foreign protein expression under aerobic condition. As indicated in Figure 3B, strains that overexpressed $y$ diS had faster growth rates than pRSF-Duet BL21 (DE3) under both static and shaking culture conditions when supplemented with $100 \mu \mathrm{g} / \mathrm{mL}$ (S)-equol. However, overexpressed $y d i T$ did not growth faster than the pRSF-Duet BL21 (DE3) when $100 \mu \mathrm{g} / \mathrm{mL}(S)$-equol was supplemented (Supplementary Figure 8). Together, these results indicated that $y$ diS gene was responsible for the equol resistance in the BL21 (ydiS) strain.

\section{Comparison of the Equol-Producing Activity of BL21 (DE3) and BL21 (ydiS)}

No equol production was detected under shaking at $200 \mathrm{rpm}$ (data not shown) and this is consistent with previous reports (Lee et al., 2016). Static culture conditions were utilized to detect the equol production in this study. In order to verify that the mutant strain BL21 (ydiS) could grow faster and provide higher yields of (S)-equol than BL21 (DE3) during fermentation, the same plasmids pETDuet-1-L-ddrc-dznr and pCDFDuet-1-L- $d h d r-t h d r$ were transformed into BL21 (DE3) [DDDT-BL21 (DE3)] and BL21 (ydiS) [DDDT-BL21 (ydis)], respectively. In $50 \mathrm{~mL}$ flasks, $20 \mathrm{~mL}$ of LB, carbenicillin (final concentration $50 \mu \mathrm{g} / \mathrm{mL}$ ), streptomycin (final concentration $50 \mu \mathrm{g} / \mathrm{mL}$ ) and two different concentrations of daidzein $(5 \mu \mathrm{g} / \mathrm{mL}$ or $50 \mu \mathrm{g} / \mathrm{mL}$ ) were added. Before adding IPTG, 
the bacterial density was adjusted to $\mathrm{OD}_{600}=0.6$. After being induced for 48 and $72 \mathrm{~h}$ under both anaerobic and aerobic conditions, the bacterial density, daidzein, and equol concentration were measured. The DDDT-BL21 ( $y$ diS) was not much better than DDDT-BL21 when $5 \mu \mathrm{g} / \mathrm{mL}$ daidzein was added as the substrate. In contrast, the growth rate, equol yield, and daidzein utilization ratio of DDDT-BL21 (ydiS) was greater than DDDT-BL21 (DE3) when $50 \mu \mathrm{g} / \mathrm{mL}$ daidzein was added as substrate both under anaerobic and aerobic conditions (Figures 4A-C; Supplementary Figures $9 \mathrm{~A}-\mathrm{C}$ ). The daidzein utilization ratio of DDDT-BL21 ( $y$ dis) reached 90\% under aerobic condition after IPTG induction, while the ratio for DDDT-BL21 was only about $27 \%$ (Figure 4C). In addition, the higher yield of $(S)$-equol for BL21 ( $y$ diS) was not only due to faster growth rates, but also because BL21 (ydiS) produced more (S)-equol than BL21 (DE3) (Figure 4D and Supplementary Figure 9D).

\section{Production of (S)-Equol Using Daidzin as the Fermentation Substrate}

As daidzin is easily collected from soybean meal, using daidzin as fermenting substrate is extremely convenient. As such, a glycoside hydrolysis gene bgl 1269 (Gang et al.,
2012) was cloned into the compatible vector pRSFDuet-1. The pRSFDuet-1-bgl plasmid was then transformed into BL21 (ydiS) [PRSF-bgl-BL21 ( $y$ diS)] to verify the gene's function. After a $72 \mathrm{~h}$ induction, daidzin or soybean meal was converted into daidzein under aerobic conditions, with the metabolites from the engineered $E$. coli had a similar HPLC peaks to the reference standard for daidzein (Supplementary Figure 10). Interestingly, after transforming pETDuet-1-L-ddrc$d z n r$, pCDFDuet-1-L- $d h d r$-thdr and pRSFDuet-1-bgl into BL21 (ydis), the transformed bacteria could not convert daidzin to $(S)$-equol (data not shown). In order to overcome this hurdle, a two-step method was utilized to transfer daidzin to $(S)$-equol. First, PRSF-bgl-BL21(ydiS) was used to convert daidzin to daidzein, then the fermentation supernatant was used as substrate for DDDT-BL21 ( $y d i S$ ) to produce $(S)$-equol. After first step fermentation, $\sim 0.17 \mathrm{mmol} / \mathrm{L}$ daidzein was produced from $50 \mu \mathrm{g} / \mathrm{mL}$ daidzin (Supplementary Figure 11A). At this point, if the water prepared for LB media was fully replaced with the fermentation supernatant, $(S)$-equol still was not detected after fermentation (data not shown). However, if $10 \%$ of the water was replaced with fermentation supernatant when preparing LB media, $\sim 0.017 \mathrm{mmol} / \mathrm{L}(S)$ equol could be detected after the two-step fermentation under both aerobic and anaerobic conditions (Supplementary Figure 11B).
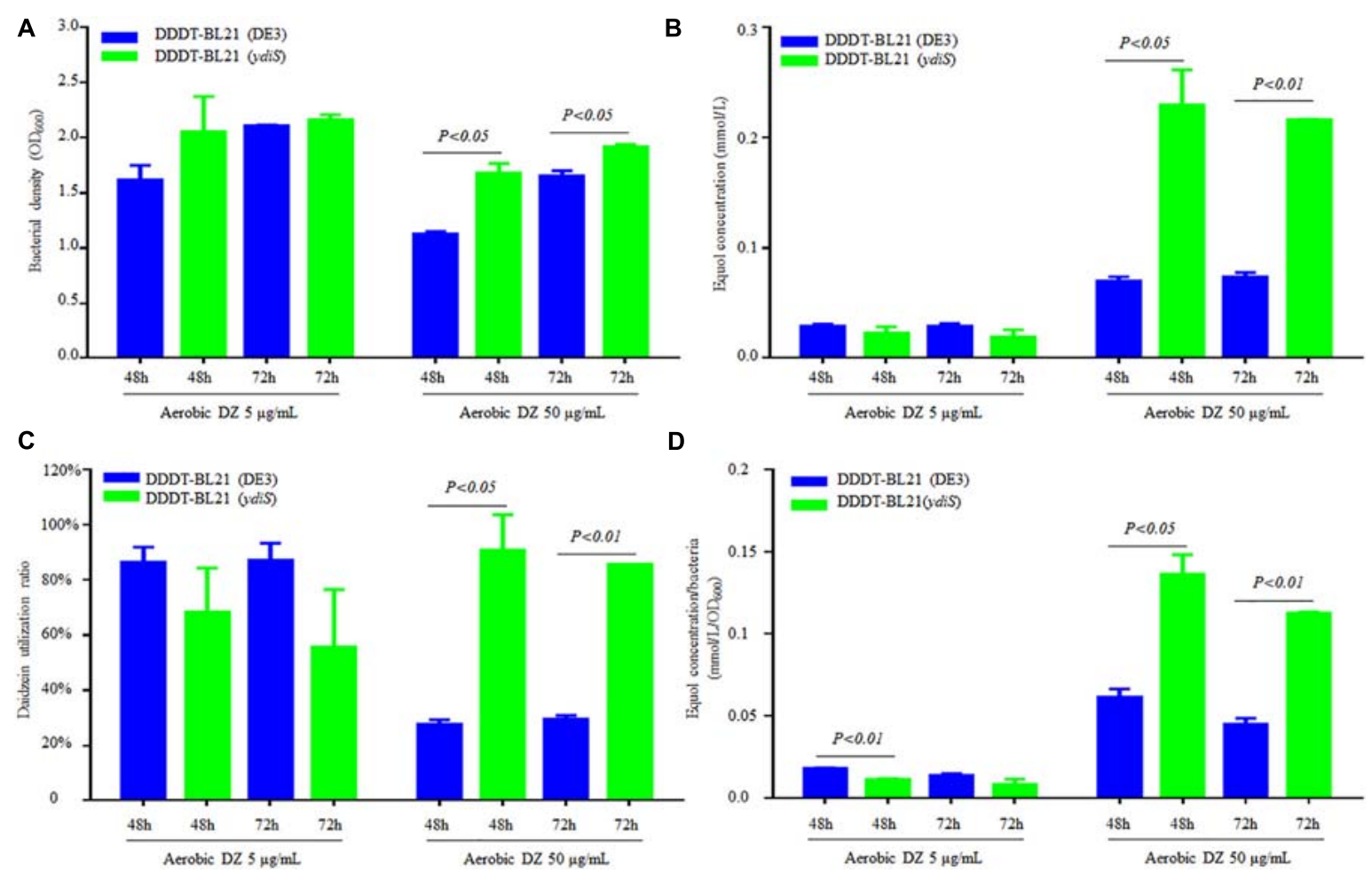

FIGURE 4 | Comparison (S)-equol production between DDDT-BL21 (ydiS) and DDDT-BL21 (DE3) under aerobic conditions. (A) Change in bacterial density; (B) Comparison of (S)-equol production of DDDT-BL21 (DE3) and DDDT-BL21 (ydiS) measured by HPLC; (C) Comparison of the daidzein utilization ratio of DDDT-BL21 (DE3) and DDDT-BL21 (ydiS); (D) Comparison of (S)-equol production per bacterium for DDDT-BL21 (DE3) and DDDT-BL21 (ydiS). At each timepoint, two duplications were measured for each sample, means and standard deviations (SD) were calculated. The Student's $t$-test was employed in this study, and $P<0.05$ was considered to be statistically significant. 


\section{DISCUSSION}

Recently, the gut microbiota has become an intensely researched topic (Garrett, 2017). The microbes in the gut have been recognized as important for proper digestive functions, allowing for a variety of dietary components to be metabolized (Koppel et al., 2017), development of the host immune system (Pamer, 2017), as well as for their impacts on some diseases and infections (Boulange et al., 2016). Dietary components, especially polyphenols have been extensively used as functional food components. Previous research has demonstrated that gut microbiota contribute to polyphenol metabolism and affect its bioavailability (Stevens and Maier, 2016); (S)-equol and soy isoflavones are typical examples. (S)-equol is the metabolite transferred from soybean meal by gut microbes (Setchell and Clerici, 2010), and the equol hypothesis infers that the main functions of soy isoflavones are due the metabolite product (S)-equol (Setchell et al., 2002). Furthermore, the safety of (S)-equol has been tested (Liu et al., 2016), indicating that further study of the function and molecular mechanism of (S)-equol is important. Heemstra et al. (2006) have developed chemical methods to synthesize (S)-equol in vitro, but natural (S)-equol obtained by microbial fermentation is more attractive, especially as it is produced in vivo. Considering the vital function of (S)-equol, its production could be important for broad applications. In this study, we constructed an engineered E. coli mutant BL21 (ydiS) that could convert higher concentration of daidzein to $(S)$-equol under aerobic conditions (Figure 4). As daidzin is the main form that is found in soybean meal, (S)-equol production from daidzin was attempted by coexpressing the genes $b g l$, L-ddrc, L-dznr, $\mathrm{L}-d h d r$, and L-thdr, ultimately producing (S)-equol using only a two-step method (Supplementary Figure 11). Coexpression of all five genes in a single system was not sufficient to convert daidzin to $(S)$-equol (data not shown). This phenomenon could have several explanations: (1) glucose generated by bgl conversion may inhibit the enzymatic activity. Not only various microbial $\beta$-glucosidases reported previously are strongly inhibited by glucose [11-13], intracellular alpha-L-rhamnosidase activity from Pseudoalteromonas sp. also affected by the monosaccharides concentration [8]. In addition, during the two-step fermentation equol could be detected $10 \%$ but not when it was fully replaced with fermentation supernatant further supporting this hypothesis. (2) host cells were too old to overexpress other genes after converting daidzin to daidzein. (3) the DDRC, DZNR, DHDR and THDR enzymes were deactivitated during the conversion of daidzin to daidzein. Regardless, the fermentation parameters and process need further adjustment.

Polyphenols have inhibitory activity on bacterial growth (Vázquez et al., 2017), which presents challenges when utilizing high yield fermentation to obtain polyphenol products (Chouhan et al., 2017). Although many antibiotic resistance genes have been identified (Liu and Pop, 2009), few studies have investigated polyphenol resistance. In this study, an (S)-equol resistant mutant was generated through a transposon mutagenesis screen. Sequencing and overexpression results revealed that $y d i$, a putative oxidoreductase gene, was responsible for $(S)$-equol resistance (Figure 3). Although Bayer et al. reported that complex I NADH oxidoreductase gene (snoD) in Staphylococcus aureus affected the susceptibility of thrombin-induced platelet microbicidal protein 1 (Bayer et al., 2006), the equol resistance mechanism of the putative oxidoreductase $y$ dis gene requires further investigation. The inhibitory effects of $(S)$-equol on bacterial growth may be due to its antioxidant function, as the potential oxidoreductase $y$ diS gene product may be counteract redox active of $(S)$-equol, thereby granting equol resistance. Schrettl et al. (2010) have reported similar phenomenon, which they hypothesize that the primary mechanism of gliotoxin inhibits Aspergillus fumigatus growth may be via antioxidant activity. Gliotoxin exposure up-regulates several antioxidant-related proteins and elevates superoxide dismutase activity. Moreover, reactive oxygen species production also increases after exposure to gliotoxin. However, glutathione (GSH) levels were significantly elevated in Aspergillus nidulans $\Delta$ gliT compared to wild-type (Carberry et al., 2012). The gliT gene encoded a gliotoxin oxidoreductase exhibits a gliotoxin reductase activity, and overexpression of GliT confers protection against exogenous gliotoxin in A. nidulans and Saccharomyces cerevisiae (Schrettl et al., 2010).

In summary, a putative oxidoreductase gene $y d i S$ was identified to be responsible for (S)-equol resistance. As a result, an engineered equol-producing bacterial strain was constructed using an (S)-equol resistant mutant [E. coli BL21 $(y d i S)]$ to coexpress the equol-synthesis genes. A two-step method was constructed to convert diadzin to $(S)$-equol under aerobic conditions, providing a new method for (S)-equol fermentation and production. In addition, the method used in this study may be useful for screening resistant host cells as an alternative method for production of anti-bacterial components, such as antibiotics and antibacterial peptides. Recently, herbal medicinal remedies have been gaining increased attention, often being combined with probiotics for therapeutic care. However, the inhibitory effects of herbs on probiotics may prevent their application; therefore, screening for polyphenol resistance genes and probiotics engineering could be beneficial for their combined use.

\section{AUTHOR CONTRIBUTIONS}

YY and XW conceived and designed the experiments. YY, HL, SM, and HC performed the experiments. YY, HL, LZ, and WL analyzed the data. YY, HL, and XW wrote the paper.

\section{FUNDING}

This study was funded by the National High Technology Research and Development Program of China (863, No. 2015AA020701), the National Nature Science Foundation (NSFC, Nos. 31100097 and 21606079), and the State Key Laboratory Breeding Base 
for Zhejiang Sustainable Pest and Disease Control (No. 2010DS700124-ZZ1604).

\section{ACKNOWLEDGMENTS}

We thank Xiaodan Wu from the Analysis Center of Agrobiology and Environmental Sciences, Institute of Agrobiology and Environmental Sciences, Zhejiang University for LC-MS analysis.

\section{REFERENCES}

Aso, T., Uchiyama, S., Matsumura, Y., Taguchi, M., Nozaki, M., Takamatsu, K., et al. (2012). A natural S-equol supplement alleviates hot flushes and other menopausal symptoms in equol nonproducing postmenopausal Japanese women. J. Womens Health 21, 92-100. doi: 10.1089/jwh.2011.2753

Axelson, M., Kirk, D. N., Farrant, R. D., Cooley, G., Lawson, A. M., and Setchell, K. D. (1982). The identification of the weak oestrogen equol [7-hydroxy3-(4'-hydroxyphenyl)chroman] in human urine. Biochem. J. 201, 353-357. doi: $10.1042 /$ bj2010353

Bayer, A. S., Mcnamara, P., Yeaman, M. N., Jones, T., Cheung, A. L., Sahl, H. G., et al. (2006). Transposon disruption of the complex I NADH oxidoreductase gene (snoD) in Staphylococcus aureus is associated with reduced susceptibility to the microbicidal activity of thrombin-induced platelet microbicidal protein 1. J. Bacteriol. 188, 211-222. doi: 10.1128/JB.188.1.211-222.2006

Blay, M., Espinel, A. E., Delgado, M. A., Baiges, I., Blade, C., Arola, L., et al. (2010). Isoflavone effect on gene expression profile and biomarkers of inflammation. J. Pharm. Biomed. Anal. 51, 382-390. doi: 10.1016/j.jpba.2009.03.028

Boulange, C. L., Neves, A. L., Chilloux, J., Nicholson, J. K., and Dumas, M. E. (2016). Impact of the gut microbiota on inflammation, obesity, and metabolic disease. Genome Med. 8:42. doi: 10.1186/s13073-016-0303-2

Carberry, S., Molloy, E., Hammel, S., O'Keeffe, G., Jones, G. W., Kavanagh, K., et al. (2012). Gliotoxin effects on fungal growth: mechanisms and exploitation. Fungal Genet. Biol. 49, 302-312. doi: 10.1016/j.fgb.2012.02.003

Chacko, B. K., Chandler, R. T., Mundhekar, A., Khoo, N., Pruitt, H. M., Kucik, D. F., et al. (2005). Revealing anti-inflammatory mechanisms of soy isoflavones by flow: modulation of leukocyte-endothelial cell interactions. Am. J. Physiol. Heart Circ. Physiol. 289, H908-H915. doi: 10.1152/ajpheart.00781.2004

Chouhan, S., Sharma, K., Zha, J., Guleria, S., and Koffas, M. A. G. (2017). Recent advances in the recombinant biosynthesis of polyphenols. Front. Microbiol. 8:2259. doi: $10.3389 /$ fmicb.2017.02259

Cobb, J. M., Mattice, J. D., Senseman, S. A., Dumas, J. A., Mersie, W., Riley, M. B., et al. (2006). Stability of pesticides on solid-phase extraction disks after incubation at various temperatures and for various time intervals: interlaboratory study. J. AOAC Int. 89, 903-912.

Cooke, G. M. (2006). A review of the animal models used to investigate the health benefits of soy isoflavones. J. AOAC Int. 89, 1215-1227.

Decroos, K., Vanhemmens, S., Cattoir, S., Boon, N., and Verstraete, W. (2005). Isolation and characterisation of an equol-producing mixed microbial culture from a human faecal sample and its activity under gastrointestinal conditions. Arch. Microbiol. 183, 45-55. doi: 10.1007/s00203-004-0747-4

Gang, L., Yang, J., Fan, X. J., and Liu, Y. H. (2012). Molecular cloning and characterization of a novel $\beta$-glucosidase with high hydrolyzing ability for soybean isoflavone glycosides and glucose-tolerance from soil metagenomic library. Bioresour. Technol. 123, 15-22. doi: 10.1016/j.biortech.2012.07.083

Garrett, W. S. (2017). Gut microbiota in 2016: a banner year for gut microbiota research. Nat. Rev. Gastroenterol. Hepatol. 14, 78-80. doi: 10.1038/nrgastro. 2016.207

Hall, M. C., O’Brien, B., and McCormack, T. (2007). Equol producer status, salivary estradiol profile and urinary excretion of isoflavones in Irish Caucasian women, following ingestion of soymilk. Steroids 72, 64-70. doi: 10.1016/j.steroids.2006. 10.010

Hall, W. L., Formanuik, N. L., Harnpanich, D., Cheung, M., Talbot, D., Chowienczyk, P. J., et al. (2008). A meal enriched with soy isoflavones increases nitric oxide-mediated vasodilation in healthy postmenopausal women. J. Nutr. 138, 1288-1292. doi: 10.1093/jn/138.7.1288
We would like to thank LetPub (www.LetPub.com) for providing linguistic assistance during the preparation of this manuscript.

\section{SUPPLEMENTARY MATERIAL}

The Supplementary Material for this article can be found online at: https://www.frontiersin.org/articles/10.3389/fmicb. 2018.01182/full\#supplementary-material

Heemstra, J. M., Kerrigan, S. A., Doerge, D. R., Helferich, W. G., and Boulanger, W. A. (2006). Total synthesis of (S)-equol. Org. Lett. 8, 5441-5443. doi: 10.1021/ ol0620444

Hwang, J., Wang, J., Morazzoni, P., Hodis, H. N., and Sevanian, A. (2003). The phytoestrogen equol increases nitric oxide availability by inhibiting superoxide production: an antioxidant mechanism for cell-mediated LDL modification. Free Radic. Biol. Med. 34, 1271-1282. doi: 10.1016/S0891-5849(03) 00104-7

Jackman, K. A., Woodman, O. L., Chrissobolis, S., and Sobey, C. G. (2007). Vasorelaxant and antioxidant activity of the isoflavone metabolite equol in carotid and cerebral arteries. Brain Res. 1141, 99-107. doi: 10.1016/j.brainres. 2007.01.007

Kinjo, J., Tsuchihashi, R., Morito, K., Hirose, T., Aomori, T., Nagao, T., et al. (2004). Interactions of phytoestrogens with estrogen receptors alpha and beta (III). Estrogenic activities of soy isoflavone aglycones and their metabolites isolated from human urine. Biol. Pharm. Bull. 27, 185-188. doi: 10.1248/bpb.27.185

Koppel, N., Maini Rekdal, V., and Balskus, E. P. (2017). Chemical transformation of xenobiotics by the human gut microbiota. Science 356:eaag2770. doi: 10.1126/ science.aag 2770

Lee, P. G., Kim, J., Kim, E. J., Jung, E., Pandey, B. P., and Kim, B. G. (2016). P212A Mutant of dihydrodaidzein reductase enhances (S)-Equol production and enantioselectivity in a recombinant Escherichia coli Whole-Cell reaction system. Appl. Environ. Microbiol. 82, 1992-2002. doi: 10.1128/AEM. 03584-15

Liu, B., and Pop, M. (2009). ARDB-Antibiotic resistance genes database. Nucleic Acids Res. 37, D443-D447. doi: 10.1093/nar/gkn656

Liu, Z., Ho, S. C., Chen, Y., Xie, Y. J., Huang, Z., and Ling, W. (2016). Research protocol: effect of natural S-equol on blood pressure and vascular function- a six-month randomized controlled trial among equol non-producers of postmenopausal women with prehypertension or untreated stage 1 hypertension. BMC Complement. Altern. Med. 16:89. doi: 10.1186/s12906-0161065-5

Low, Y. L., Taylor, J. I., Grace, P. B., Dowsett, M., Scollen, S., Dunning, A. M., et al. (2005). Phytoestrogen exposure correlation with plasma estradiol in postmenopausal women in European prospective investigation of cancer and nutrition-norfolk may involve diet-gene interactions. Cancer Epidemiol. Biomarkers Prev. 14, 213-220.

Marrian, G. F., and Haslewood, G. A. (1932). Equol, a new inactive phenol isolated from the ketohydroxyoestrin fraction of mares' urine. Biochem. J. 26, 1227-1232. doi: 10.1042/bj0261227

Nielsen, I. L., and Williamson, G. (2007). Review of the factors affecting bioavailability of soy isoflavones in humans. Nutr. Cancer 57, 1-10. doi: 10.1080/01635580701267677

Oyama, A., Ueno, T., Uchiyama, S., Aihara, T., Miyake, A., Kondo, S., et al. (2012). The effects of natural S-equol supplementation on skin aging in postmenopausal women: a pilot randomized placebo-controlled trial. Menopause 19, 202-210. doi: 10.1097/gme.0b013e318227427b

Ozasa, K., Nakao, M., Watanabe, Y., Hayashi, K., Miki, T., Mikami, K., et al. (2005). Association of serum phytoestrogen concentration and dietary habits in a sample set of the JACC Study. J. Epidemiol. 15(Suppl. 2), S196-S202. doi: $10.2188 /$ jea.15.S196

Pamer, E. G. (2017). Microbial tuning of the mammalian immune system. Trends Mol. Med. 23, 379-380. doi: 10.1016/j.molmed.2017.03.006

Rufer, C. E., and Kulling, S. E. (2006). Antioxidant activity of isoflavones and their major metabolites using different in vitro assays. J. Agric. Food Chem. 54, 2926-2931. doi: 10.1021/jf053112o 
Saltikov, C. W., and Newman, D. K. (2003). Genetic identification of a respiratory arsenate reductase. Proc. Natl. Acad. Sci. U.S.A. 100, 10983-10988. doi: 10.1073/ pnas. 1834303100

Sarkar, F. H., and Li, Y. (2003). Soy isoflavones and cancer prevention. Cancer Invest. 21, 744-757. doi: 10.1081/CNV-120023773

Schrettl, M., Carberry, S., Kavanagh, K., Haas, H., Jones, G. W., O’Brien, J., et al. (2010). Self-protection against gliotoxin-a component of the gliotoxin biosynthetic cluster, GliT, completely protects Aspergillus fumigatus against exogenous gliotoxin. PLoS Pathog. 6:e1000952. doi: 10.1371/journal.ppat. 1000952

Setchell, K. D., Brown, N. M., and Lydeking-Olsen, E. (2002). The clinical importance of the metabolite equol-a clue to the effectiveness of soy and its isoflavones. J. Nutr. 132, 3577-3584. doi: 10.1093/jn/132.12.3577

Setchell, K. D., and Clerici, C. (2010). Equol: history, chemistry, and formation. J. Nutr. 140, 1355S-1362S. doi: 10.3945/jn.109.119776

Setchell, K. D., Clerici, C., Lephart, E. D., Cole, S. J., Heenan, C., Castellani, D., et al. (2005). S-equol, a potent ligand for estrogen receptor beta, is the exclusive enantiomeric form of the soy isoflavone metabolite produced by human intestinal bacterial flora. Am. J. Clin. Nutr. 81, 1072-1079. doi: 10.1093/ajcn/ 81.5.1072

Setchell, K. D., and Cole, S. J. (2006). Method of defining equol-producer status and its frequency among vegetarians. J. Nutr. 136, 2188-2193. doi: 10.1093/jn/ 136.8.2188

Shimada, Y., Takahashi, M., Miyazawa, N., Abiru, Y., Uchiyama, S., and Hishigaki, H. (2012). Identification of a novel dihydrodaidzein racemase essential for biosynthesis of equol from daidzein in Lactococcus sp. strain 20-92. Appl. Environ. Microbiol. 78, 4902-4907. doi: 10.1128/AEM.00410-12

Shimada, Y., Takahashi, M., Miyazawa, N., Ohtani, T., Abiru, Y., Uchiyama, S., et al. (2011). Identification of two novel reductases involved in equol biosynthesis in Lactococcus strain 20-92. J. Mol. Microbiol. Biotechnol. 21, 160-172. doi: $10.1159 / 000335049$
Stevens, J. F., and Maier, C. S. (2016). The chemistry of Gut microbial metabolism of polyphenols. Phytochem. Rev. 15, 425-444. doi: 10.1007/s11101-016-9459-z

Turner, R., Baron, T., Wolffram, S., Minihane, A. M., Cassidy, A., Rimbach, G., et al. (2004). Effect of circulating forms of soy isoflavones on the oxidation of low density lipoprotein. Free Radic. Res. 38, 209-216. doi: 10.1080/ 10715760310001641854

Vázquez, L., Flórez, A. B., Guadamuro, L., and Mayo, B. (2017). Effect of soy isoflavones on growth of representative bacterial species from the human Gut. Nutrients 9:727. doi: 10.3390/nu9070727

Withers, T. R., Yin, Y., and Yu, H. D. (2014). Identification of novel genes associated with alginate production in Pseudomonas aeruginosa using mini-himar1 mariner transposon-mediated mutagenesis. J. Vis. Exp. 10:85. doi: $10.3791 / 51346$

Wong, S. M., and Mekalanos, J. J. (2000). Genetic footprinting with marinerbased transposition in Pseudomonas aeruginosa. Proc. Natl. Acad. Sci. U.S.A. 97, 10191-10196. doi: 10.1073/pnas.97.18.10191

Xiao, Y., Zhang, S., Tong, H., and Shi, S. (2017). Comprehensive evaluation of the role of soy and isoflavone supplementation in humans and animals over the past two decades. Phytother. Res. 32, 384-394. doi: 10.1002/ptr.5966

Conflict of Interest Statement: The authors declare that the research was conducted in the absence of any commercial or financial relationships that could be construed as a potential conflict of interest.

Copyright (C) 2018 Li, Mao, Chen, Zhu, Liu, Wang and Yin. This is an open-access article distributed under the terms of the Creative Commons Attribution License (CC BY). The use, distribution or reproduction in other forums is permitted, provided the original author(s) and the copyright owner are credited and that the original publication in this journal is cited, in accordance with accepted academic practice. No use, distribution or reproduction is permitted which does not comply with these terms. 